\title{
Reflexión transdisciplinaria acerca del deber ser de la gestión escolar en Venezuela
}

\author{
Pulido, Sirse* \\ Siracusa, Giuseppe** \\ Ardiles, Arlene***
}

\begin{abstract}
Resumen
La gerencia en las escuelas bolivarianas como todo proceso humano está axiomáticamente unido a la evolución de la sociedad, en tanto que es una sucesión de acontecimientos históricos praxológicos, signados por la intervención de distintos enfoques gerenciales entremezclados, formando un entramado o bootstrap transdisciplinar, un todo unificado sobre la gestión escolar participativa. Bajo este contexto, el presente artículo tiene como objetivo analizar los diferentes enfoques epistemológicos y teóricos desde la perspectiva transdisciplinaria de la gestión escolar participativa en las escuelas bolivarianas, fundamentado en una revisión documental. Destaca como resultado que las exigencias educativas implican, la intervención transdisciplinaria, ya que no se abordan aspectos aislados dentro del contexto escolar. Por lo que se concluye que la gestión escolar en el marco de las escuelas bolivarianas tienen el compromiso de involucar a los principales actores del hecho educativo e interconectarlos con las responsabilidades establecidas en el Proyecto Educativo Nacional y su entorno institucional.
\end{abstract}

Palabras clave: Gestión escolar participativa, transdisciplinaridad, escuelas bolivarianas.

Recibido: 28-10-10. Aceptado: 30-05-11

* Licenciada en Educación. Especialista en Gerencia Pública. Doctorante en Ciencias Gerenciales de la UNEFA-Núcleo Yaracuy. Postdoctorante en Educación Latinoamericana en la UPELRIEAC. Docente a tiempo convencional de la UNEFA-Núcleo Yaracuy. Venezuela.

E-mail: sirsepulido78@hotmail.com.

** Ingeniero Civil. Especialista en Gerencia Pública. Doctorante en Ciencias Gerenciales de la UNEFA-Núcleo Yaracuy. Postdoctorante en Educación Latinoamericana en la UPEL-RIEAC. Docente a dedicación exclusiva de la UNEFA-Núcleo Yaracuy. Venezuela.

E-mail: siracusa25@gmail.com.

*** Arquitecta. Especialista en Gerencia Pública. Doctorante en Ciencias Gerenciales de la UNEFA-Núcleo Yaracuy. Docente a tiempo convencional de la UNEFA-Núcleo Yaracuy. Venezuela. E-mail: arleneardiles@gmail.com. 


\title{
A Transdisciplinary Reflection about What School Management in Venezuela Ought to be
}

\begin{abstract}
Management in Bolivarian schools, like any human process, is axiomatically linked to the evolution of society, in that it is a succession of historical-praxiological events marked by the intervention of different, intermingled management approaches, forming a lattice or transdisciplinary "bootstrap," a unified whole regarding participatory school management. In this context, the objective of this article is to analyze the different epistemological and theoretical approaches from a transdisciplinary perspective of participative school management in Bolivarian schools, based on a documentary review. One outstanding result is that educational demands imply transdisciplinary intervention, since they do not deal with isolated aspects in the school context. Therefore, conclusions are that school management within the framework of the Bolivarian schools has the commitment to involve the main actors in the educational act and interconnect them with the responsibilities established in the National Educational Project and its institutional environment.
\end{abstract}

Key word: Participatory school management, transdisciplinarity, Bolivarian schools.

\section{Introducción}

La actual reforma del sistema educativo venezolano, iniciada con la creación de las Escuelas Bolivarianas por el Ministerio de Educación Cultura y Deporte (2000), origina una serie de expectativas en relación con las modificaciones formuladas. Estas conllevan a un cambio en la organización de la institución escolar, promoviendo un modelo curricular flexible y abierto, que permita hacer las adecuaciones curriculares conjuntamente con el diseño de Proyecto Educativo Integral Comunitario (P.E.I.C), en atención a las necesidades e intereses de los educandos; a los problemas socioeconómicos, culturales y ambientales que se dan en su entorno y a los avances en el campo de la psicopedagogía.

Esta educación actualmente plantea la necesidad de un nuevo tipo de director, de personal docente, de miembros de la comunidad educativa más participa- tivos a tono con la creciente autonomía de los centros educativos, orientados a mejorar los estándares de calidad y contenido que aseguren el desarrollo de conocimiento y competencia en los estudiantes.

En esta línea del pensamiento, es incuestionable que las reformas educativas contemplan cambios en la dirección de las instituciones escolares, esencialmente en lo que respecta a la praxis de la función directiva; por cuanto se le está transfiriendo mayores atribuciones a los centros educativos, ya sea en el orden social, curricular, administrativo, financiero y comunitario lo que supone competencias y habilidades particulares en el director.

Habida cuenta de esta realidad fenoménica el propósito del articulo se inscribe en lo más profundo de la esencia de la metamorfosis educativa venezolana en el entendido que aportar conocimientos en este sentido conlleva a indagar, compren- 
Reflexión transdisciplinaria acerca del deber ser de la gestión escolar en Venezuela Pulido, Sirse; Siracusa, Giuseppe y Ardiles, Arlene

der y explicar estrategias para reconstruir la gestión escolar participativa en las Escuela Bolivarianas, lo que sin duda amerita una nueva perspectiva teórica y praxológica en aras de regenerar su práctica y proporcionarle a los gerentes educativos otra perspectiva para su hacer gerencial en consonancia con los principios de participación inscritos en los postulados de la Constitución de la República Bolivariana de Venezuela, el Proyecto Educativo Nacional y los Lineamientos de las Escuelas Bolivarianas, desde una visión compartida y transdisciplinaria.

Asimismo, el conocimiento generado constituirá un replanteamiento ante una circunstancia histórica, a sabiendas de que este conocimiento, siempre será traducción y reconstrucción. Asimismo el conocer y pensar en la gestión de las escuelas bolivarianas no es llegar a una verdad totalmente cierta, es dialogar con la certidumbre-incertidumbre de este evento, con miras a dejar una postura teórica que ha de ser útil en la medida en que se adapte a las situaciones particulares del contexto.

\section{Aproximación a la realidad fenoménica de la gestión escolar participativa}

En las instituciones educativas la gestión gerencial evoluciona en tanto que es una sucesión de acontecimientos históricos-praxológicos, es un haz de procesos y un devenir en continúa metamorfosis que en su desplazamiento se transposiciona con la intervención de enfoques disciplinarios que se trastocan y funden para dar cuenta de su estado.
Siendo así, es casi natural pensar que si en el hacer gerencial intervienen factores de distintas ciencias: psicológicas, tecnológicas, sociológicas, humanistas entre otras, las cuales categóricamente le dan significado a las actividades del quehacer humano, entonces el paradigma positivista pareciera no ser el más adecuado para comprender y generar repuestas a las múltiples exigencias propias de la vida organizacional, puesto que a manera de lentes sólo nos permiten ver, como lo expresa Lakatos (1982), lo que está dentro de los parámetros de la racionalidad instrumental. Así pues, el entramado o bootstrap de la realidad, complicada telaraña de relaciones entre las diversas partes de un todo unificado, es preciso explorarlo, valiéndonos de distintos prismas a sabiendas que el ser humano no puede conocer haciendo un retrato fiel y exacto del exterior sino que todo conocimiento como señala Morín (2003) es una traducción de la realidad externa y al decir de Platón en su célebre Mito de las Cavernas, conoceremos de esa realidad física sólo su sombra.

Ciertamente, la educación y específicamente la gestión escolar tiene una responsabilidad sin precedentes en la actual coyuntura caracterizada por la globalidad de los saberes, la vorágine de cambios que vivimos en los albores del siglo $X X I$ y la necesidad de reflexionar sobre la identidad -con cabeza global y pies locales- para no caer en los peligros del economicismo y de las incertidumbres a ultranza; en definitiva requiere de nuestro conocimiento y decisiones con un contenido ético que coadyuve al logro de convivir garantizando la existencia y vida planetaria para el porvenir. Ideal alcanzable 
que no se cumplirá si no hacemos nuestro mayor esfuerzo para convertirlo en una realidad en proceso de construcción. Sobre este aspecto Morín (2001:63) afirma: "...el problema es que tenemos un mundo rico que acaba de llegar a su fin sin que todavía haya nacido un mundo nuevo...". Con todo, ese mundo nuevo comienza a visualizarse, someramente es verdad, pero el hecho es que está ocurriendo.

Así por ejemplo, el informe mundial sobre el futuro de la educación, de la Organización de las Naciones Unidas para la Educación (UNESCO, 2005) señala la perspectiva de una expansión del acceso a la educación y la continuación de rápidos cambios en el medio ambiente económico, social y cultural, por lo que se prevé que aumente la preocupación por la calidad de la escolarización y en particular por los resultados obtenidos en el proceso de aprendizaje. Las opiniones en cuanto a los sectores prioritarios de intervención divergen mucho; la tendencia ha sido en centrar el interés en los currículos escolares, en modificaciones del contenido programático, innovaciones en el proceso enseñanza-aprendizaje, lo cual ha tenido como consecuencia transformar el papel del profesor y otorgar nuevas responsabilidades al estudiante.

Esta renovación de contenidos, métodos y currícula han complejizado el papel del profesional de la docencia que de hecho lo es ya que la educación en la época actual, en momentos de post-industrialización, posee un nuevo significado. El Informe de la UNESCO para la educación del siglo XXI (2001) nos presenta un giro copernicano en lo que respecta a la concepción didáctico-pedagógica-ge- rencial, al sostener que el reto del aula, del docente y en consecuencia del gerente educativo es el aprender a conocer; a hacer, a convivir y a ser; esto supone que los docentes deben mediar los aprendizajes y gerenciar las escuelas desde estas cuatro perspectivas, superando las visiones reductivas, cognitivas e integrando aspectos vitales y existenciales como lo son las habilidades, la convivencia, lo social y la ética.

En el contexto nacional, es innegable que en las últimas décadas el sistema educativo venezolano ha incorporado reformas profundas en la búsqueda incesante de mejorar la calidad de la educación en beneficio del colectivo. Es así como, el Ministerio de Educación Cultura y Deportes (2000), concretiza la política de transformación de la calidad educativa a través del fortalecimiento de la gestión pedagógica, creando las Escuelas Bolivarianas según la Resolución $N^{\circ} 179$ de fecha 15 de Septiembre de 1999, constituyendo según lo expresado por (Ponce, 1999:32), el eje dinamizador del proceso escolar, "desde las cuales se promoverán experiencias innovadoras con el fin de accionar dos ejes esenciales: a) elevar el rendimiento académico y b) garantizar la educación del educando con sentido humanístico y social...".

Partiendo de los planteamientos hasta ahora formulados, se asume que el proyecto de Escuelas Bolivarianas demanda una nueva forma de gestión gerencial participativa y, en consecuencia, de herramientas de planificación, donde el personal docente, directivos en general y el director en particular deberán adecuar su desempeño hacia el logro de los fines objetivos y metas de la institución en 
Reflexión transdisciplinaria acerca del deber ser de la gestión escolar en Venezuela Pulido, Sirse; Siracusa, Giuseppe y Ardiles, Arlene

consonancia con los altísimos propósitos de la educación bolivariana, cuya visión se centra en construir un nuevo modelo de sociedad solidaria, participativa y protagónica, imprescindible para salvar el destino de la familia humana, acentuando el papel del trabajo gerencial integrado por el seguimiento a los procesos pedagógicos y la ejecución de innovaciones organizacionales en aras de alcanzar tan alto cometido.

Insertado en este panorama, surge el Curriculum Básico Nacional (CBN, 1997) como propuesta ante la necesidad de concertar con las grandes aspiraciones de la mayoría de los países del globo, la forma de lograr una sociedad posmoderna basada en la confianza de que la institución escolar es el ente capaz de trasformar al mundo a través de la formación de los nuevas ciudadanos.

Posteriormente, esta propuesta nacional buscó su concreción en el año 2001 a través del Proyecto Educativo Nacional (PEN), cuyo objetivo según el Ministerio de Educación, Cultura y Deportes (2001) constituye el puente para dejar atrás definitivamente el segundo milenio y comenzar el nuevo con un espíritu más fraterno y más consciente del entorno social y ambiental; basado en asumir, por nombrar algunos aspectos, una configuración humanista y cooperativa, formar en la cultura de la participación ciudadana, de la solidaridad social y el diálogo intercultural, las transdisciplinaridad del saber y formación en valores ciudadanos.

De esta forma, la prioridad de la agenda educativa, en los próximos años, tendrá que estar dirigida no sólo hacia las cuestiones relacionadas con los procesos de construcción del conocimiento-a- prendizaje y de gestión escolar, sino también a las cuestiones afectas a la sustentabilidad ecológica, a la ciudadanía planetaria como consecuencia de la evolución del pensamiento, de la inteligencia y de la conciencia humana en una perspectiva integrada. O sea, la prioridad educativa tendrá que también estar dirigida a las preguntas relativas a la interculturalidad, a la diversidad y al desarrollo pleno de la conciencia humana, dimensiones que el gerente escolar está llamado a conocer y reflexionar, pues la diversidad sustentable es, sin duda, una de las grandes preocupaciones de nuestro tiempo; en esencia, para la democracia fundada en la complejidad de las interacciones entre unidad y diversidad y en la comprensión de las nuevas identidades que surgen en una dinámica de naturaleza compleja.

Con esta visón es indudable que, coincidiendo con los planteamientos de Limardo (2000), la conducción de las instituciones educativas, demanda de los directores competencias y exigencias académicas, una gama de habilidades personales, profesionales y técnicas que deberán ser adquiridas y perfeccionadas a través de un sistemático y permanente proceso de actualización y metamorfización, con el propósito de alinearse a los nuevos acontecimientos científicos.

En este nuevo proyecto, el gerente se constituye en el ente dinamizador para conectar la escuela con la comunidad educativa, la cual está concebida como una organización primordial en el proceso formativo, al sumar a todos los sectores e instituciones de índole social que se identifican con la comunidad local. Al respecto el (Ministerio de Educación, 1996:7), en el manual sobre comunidad educativa destaca: 
"La participación activa de los individuos en la tarea educativa y en las labores de la comunidad local, conlleva a un proceso continuo y ascendente que garantiza la integración de la escuela con la comunidad. Este proceso implica que la escuela debe integrarse a los programas de desarrollo de la comunidad, y a la vez actuar como centro de promoción de la misma. La escuela tendrá como función básica, la de auspiciar la participación, cooperación y la integración en los miembros de su comunidad".

Desde este ángulo visionario, es importante destacar que para cualquier cambio trascendente en la vida escolar, el director juega un papel cardinal por cuanto debe dirigir, promover y orientar la gestión de la institución a fin de lograr estos objetivos trascendentales en la construcción de la nueva escuela que se aspira con la creación de las Escuelas Bolivarianas.

\section{El continuo histórico en el devenir de los saberes: de la disciplinaridad a la transdisciplinaridad.}

En el recorrido o continuo histórico que ocupa esta investigación, es conveniente comenzar por destacar que en el siglo XII, la raíz disciplinar surgió con la aparición de las tempranas Universidades como Salerno, Bologna, Paris, Oxford y Cambridge, partiendo sólo de las facultades de Medicina, Filosofía, Teología y Derecho, fueron las primeras en impulsar la disciplinaridad. En torno a esas cuatro áreas se reunía la totalidad del conocimiento, y de hecho, los académicos eran versátiles y omniscientes, dignos antecesores del hombre renacentista.
En este continuo, surgió la interdisciplinaridad la cual constituyó una manifestación en avance durante el siglo $X X$, que nos obliga a recorrer un largo camino que podría dividirse en tres momentos importantes. El primero marcaría su surgimiento y se inicia con el pensamiento occidental, desde los clásicos de la antigua Grecia hasta los pensadores contemporáneos. En esta etapa, en el año 1637, la obra de Descartes, El discurso del método, con la res-cogitans, cosa que piensa y la res extensa, cosa medible, describe el primer planteamiento de la división sujeto y objeto, el elemento que origina la variedad de las disciplinas y que puede tomarse como punto de partida. El segundo momento podría ubicarse desde la primera guerra mundial hasta los años 30 con esfuerzos aislados sin connotación en el mundo académico; el tercero, desde finales de la segunda guerra mundial hasta el presente.

En este continuo la interdisciplinaridad se instituyó epistemológicamente como reagrupación de saberes que gracias a la intervención de la sociedad científica interdisciplinaria se le añadió a la necesidad de la comunicación entre las disciplinas; fueron exponentes de estas ideas: Gottfried Wilhelm von Leibnitz y Jean Amos Komenski (Comenio). Este último señala Gusdorf (1983), propuso la pansophia, como pedagogía de la unidad, capaz de eliminar la fragmentación del saber de las disciplinas.

En el siglo XX el campo de las ciencias vivió momentos impactantes, entre ellos las dos guerras mundiales y esto lleva a plantearse nuevas expectativas, sobre todo en el marco de las ciencias sociales. La complejidad del momento obli- 
Reflexión transdisciplinaria acerca del deber ser de la gestión escolar en Venezuela Pulido, Sirse; Siracusa, Giuseppe y Ardiles, Arlene

gó a dar soluciones multidisciplinarias a dichos acontecimientos en cuya finalidad los problemas se descomponían en espacios más pequeños unidisciplinarios, donde se agregaban soluciones parciales a la solución integral. La multidisciplinariedad desde el punto de vista etimológico significa muchas disciplinas, es decir, varias disciplinas abordando el mismo objeto de estudio pero sin interconexión alguna o relación aparente entre ellas. No obstante, el surgimiento del enfoque sistémico de Bertalanffy (1984) llevó a que los estudios multidisciplinarios no suplieran todas las expectativas, y esto, a su vez, condujo a la aparición de las investigaciones interdisciplinarias que entendían los problemas en su totalidad pero visto desde diferentes disciplinas.

Después de la segunda guerra mundial, en el tercer período de evolución de la interdisciplina, los propios problemas mundiales obligaron a su desarrollo. Se inició entonces lo interdisciplinar en el mundo la cooperación en las áreas económicas, políticas-científicas y culturales.

La aparición de la UNESCO como organización para la cooperación internacional impulsó al desarrollo interdisciplinario. Su propósito central es la transferencia de métodos de una disciplina a otra. Aquí al igual que en la multidisciplinariedad se sobrepasa el campo de los saberes específicos.

A finales de los años 1960, Peñuela (2005) reseña que la UNESCO promovió el trabajo desde perspectivas interdisciplinarias en aras de solucionar los problemas fundamentales del momento y fue cuando surgieron los grandes representantes en el campo ciencias sociales y humanas, entre ellos Piaget, Mackenzie, La- zarsfeld Gusdorf, Smirnov, Morín y otros quienes embebidos por la nueva exigencia de la sociedad y de la ciencia crearon un movimiento internacional a favor de las investigaciones transdisciplinarias.

Sobre la base de esta narración histórica, es posible señalar que la transdisciplinariedad es una concepción mucho más reciente, y quizás la propia complejidad del mundo en que vivimos nos obliga a valorar los fenómenos desde la perspectiva de la interconexión; pues es sabido aún en la propia cotidianidad, que las actuales situaciones físicas, biológicas, sociales y psicológicas interactúan autopoiética y recíprocamente.

Cabe destacar que la transdisciplinaridad como movimiento académico e intelectual se desarrolló significativamente durante los últimos 15 años, a pesar de que es un fenómeno que surgió a partir de los nuevos cuestionamientos filosóficos de la ciencia del siglo XX frente al la corriente positivista. Lo transdisciplinario tiene como intención superar la fragmentación del conocimiento, más allá del enriquecimiento de las disciplinas con diferentes saberes multidisciplinares y del intercambio epistemológico y de métodos científicos de los saberes interdisciplinares. Lo que caracteriza a la transdisciplinariedad no es sólo la realidad interactuante sino su totalización.

A pesar de ser un fenómeno de la actualidad, Platón en sus célebres diálogos expresaba: "Si encuentro a alguien que sea capaz de ver la realidad en su diversidad y, al mismo tiempo, en su unidad, ese es el hombre al que yo busco como a un Dios". Esta afirmación, constituye una valoración entre dos niveles de realidad: el de la disciplinariedad como si- 
nónimo de diversidad de saberes y el de la transdisciplinariedad como saber global ante una realidad que desde todo punto de vista es integradora de allí que al decir de Morín (2003:52):

"...por todas partes, se es empujado a considerar, no los objetos cerrados y aislados, sino como sistemas organizados en una relación coorganizadora con su entorno (...); por todas partes se sabe que el hombre es un ser físico y biológico, individual y social, pero en ninguna parte puede instituirse una ligazón entre los puntos de vista físico, biológico, antropológico, psicológico, sociológico. Se habla de interdisciplinariedad, pero por todas partes el principio de disyunción sigue cortando a ciegas".

En efecto, estamos en el renacimiento de un nuevo paradigma del saber, al que llamamos el paradigma polisistémico del saber, en el cual existe una ligazón de conocimientos concéntricos de nunca acabar en el cual se dan la mano y ocasionalmente se abrazan los postulados de Descartes, Newton, Galileo, Morín, Luckman, Beyman, Brenteno y otros, integrándose en un contexto mucho más amplio, emergente y de mayor sentido que nos obliga como dice Martínez (2006) a adoptar una metodología transdisciplinaria para poder captar la riqueza de la interacción entre los diferentes subsistemas que estudian las disciplinas particulares.

En suma, la transdisciplinariedad es un concepto complejo que desde el punto de vista etimológico del término subyace una pluridimensionalidad, ya que su vocablo está conformado por la preposición latina "trans" y el sustantivo adjetivado "disciplinariedad". El prefijo "trans", que significa "más allá y a través de", se utiliza predominantemente para indicar eventos en los que no existen fronteras entre las disciplinas, es decir, las acciones que se mueven dentro y a través de una determinada disciplina.

Así nos referimos a una nueva comprensión de la realidad, a la construcción del conocimiento y del saber, a la metodología o forma de conocer desde la teoría del caos y la complejidad y una actitud ante el mundo y la vida que nos lleva a cosmovisonar la gestión escolar participativa de las Escuelas Bolivarianas de una manera amplia, profunda y reticular. En este orden de ideas, la trandisciplinariedad nos habla de lo que está al mismo tiempo entre, a través de y más allá de las disciplinas, siendo su finalidad en este estudio la comprensión del mundo y, en consecuencia, la gestión escolar desde la articulación de las diferentes áreas de conocimiento y saberes.

Significa entonces, que la transdisciplinariedad, según Nicolescu (1998), actual director del Centre International des Recherches et Études Transdisciplinaires (CIRET), en el Simposio Internacional sobre Transdisciplinariedad, organizado por la UNESCO en Mayo de 1998, afirma es un término inventado en su momento para expresar, sobre todo en el campo de la enseñanza, la necesidad de una feliz transgresión de las fronteras entre las disciplinas, de una superación de la pluri y de la interdisciplinariedad; que en el PEN se concretiza mediante la transversalidad y globalización de los aprendizajes, de modo que los límites de las disciplinas individuales se trasciendan para tratar problemas desde perspectivas múltiples con vista a generar conocimiento emergente mediante la inte- 
Reflexión transdisciplinaria acerca del deber ser de la gestión escolar en Venezuela Pulido, Sirse; Siracusa, Giuseppe y Ardiles, Arlene

gración y transformación de perspectivas gnoseológicas distintas.

En consecuencia, para Nicolescu (1996:5):

"la disciplinariedad, la pluridisciplinariedad, la interdisciplinariedad y la transdisciplinariedad son las cuatro flechas de un solo y mismo arco: el del conocimiento".

La clave de la bóveda de la transdisciplinariedad reside en la racionalidad abierta, que trasciende el dominio de las ciencias exactas por su diálogo y su reconciliación, no solamente con las ciencias humanas sino también con el arte, la literatura, la poesía y la experiencia interior.

La perspectiva transdisciplinaria plantea estrategias de largo alcance, incluye enfoques y teorías que en conjunto convoca a reflexionar en torno a la gestión escolar participativa en las escuelas bolivarianas a la luz de la normativa legal actual, en los cuales la teorización, la intuición, el compromiso social y la proposición de cambios profundos, constituyen la brújula de los autores, con la intención de unificar conocimientos, superando trincheras que tradicionalmente delimitan las posturas académicas, procurando que los saberes de los campos dispares se integren en una visión de conjunto permitiendo estudiar sus conexiones y relaciones de coordinación y subordinación.

Desde esta ángulo visionario, Bourdieu (1995) expresa la perspectiva transdisciplinaria nos hacer ver el mundo y la realidad de otra manera, lo que invita a plasmar un pensamiento sistémico, integral, holístico, multidisciplinario, interdisciplinario $y$, finalmente transdisciplinario, ante la necesidad de concebir una gestión escolar que pueda dar respuestas viables, confiables y con equidad a los planteamientos de la realidad venezolana, esta primera aproximación representa el primer arco del conocimiento sobre el objeto de estudio.

\section{El otro arco del conocimiento: gerencia social, gerencia participativa y gestión escolar}

Para transversalizar el recorrido por este arco, es oportuno mencionar que generalmente se define la gerencia como la gestión de una organización dirigida a organizar las tareas, a fin de que los integrantes puedan cumplirlas con métodos, visión y comprensión llevándolas a buenas posibilidades de éxito. Con base a esta concepción, la gerencia puede ser directiva, el gerente da órdenes y controla todo el proceso; consultiva, el gerente mantiene el control y consulta a sus colaboradores; por delegación, el control del gerente es bajo y su intervención poca y participativa en la cual el control del gerente es bajo y su intervención y la de los colaboradores es alta; para ello se requiere de un equipo con actitud de integración y corresponsabilidad, con sentido de pertenencia o identidad que permita el trabajo en equipo y empoderamiento de los partícipes de la gestión, lo cual conlleva al mejoramiento en la calidad de las decisiones; el aumento de la productividad; moral laboral más alta, menores rotaciones, atrasos y ausencias así como la mejor comunicación y resolución de conflictos en la organización.

Enmarcada en este contexto del segundo arco del conocimiento, la gerencia social para Kliksberg (1998:78): 
"es una operación organizacional de índole diferente, fluida, de composición interorganizacional que permite enfrentarse a situaciones muy particulares y subjetivas relacionadas con problemas sociales de una determinada comunidad".

El autor plantea que la gerencia social eficiente tiende a optimizar el rendimiento de los esfuerzos de los actores frente a los grandes déficits sociales y el mejoramiento del funcionamiento de la comunidad o de la organización, en que la inversión en capital humano y capital social, representado en este estudio por sus actores y su impacto socioeducativo desde la Escuelas Bolivarianas en la sociedad, representan los elementos esenciales de la gerencia participativa que centrada en los postulados del PEN tenga como horizonte una teoría capaz de articular distintas disciplinas para proveer un fundamento sólido que posibilite la praxología de la gestión escolar participativa.

Cabe destacar que la Gerencia Social, se ubica en la intersección de tres áreas: desarrollo social, políticas públicas y gerencia pública. Los aportes de estas tres disciplinas a su desarrollo, proporcionan un enfoque integral y con un amplio espectro de posibilidades para generar una teoría como la presente. Pues, la articulación de estas disciplinas tocará por igual a los actores, la participación ciudadana y las políticas estadales que norman tal interrelación.

En esta línea transversal del pensamiento, en relación a la gerencia participativa se considera conveniente citar a Piaget (2001), el famoso psicólogo francés, quien declaro que, el objeto principal de la educación es por una parte crear hombres que sean capaces de hacer co- sas nuevas, no repetir simplemente lo que han hecho las otras generaciones, hombres que sean creativos, inventivos y descubridores; y por la otra conformar mentes que puedan ser críticas, que puedan verificar y no aceptar a ultranzas todo los que se les ofrece.

Es así como, la gestión participativa se inspira en el nuevo modelo de gestión abordado en los estudios realizados sobre el rendimiento y la satisfacción del personal que hace vida en las organizaciones. Estos estudios, entre los cuales destaca el de Aktouf (1998) han revelado que aquellas organizaciones basadas en modelos de gestión participativa muestran un rendimiento y una satisfacción del personal mayor que en modelos de dirección unipersonal. Esto ha ocasionado que los modelos de gestión tradicionales sean cuestionados existiendo la necesidad de reorientarlos. Esta nueva visión de administración ha repercutido directamente en el plano educativo, pues parece ser, que no se tendrá una cultura de participación si esta no es defendida, asumida y procesada en la escuela.

El fortalecimiento de una cultura de participación de los distintos actores con la gestión escolar, teniendo como eje el acercamiento a la escuela, trae consigo el consecuente mejoramiento de la calidad de la docencia y por ende, el de la propia gerencia. Pues como dice Schemelkes (1992:31):

"En una escuela, quizás más que en ningún tipo de organización, los resultados dependen de las personas y de las interrelaciones entre las personas. $Y$ en una escuela al igual que cualquier organización todas las actividades están estrechamente vinculadas entre sí. Por 
Reflexión transdisciplinaria acerca del deber ser de la gestión escolar en Venezuela Pulido, Sirse; Siracusa, Giuseppe y Ardiles, Arlene

eso, un movimiento hacia una mejor calidad del proceso educativo requiere el involucramiento activo de todos los agentes implicados. No obstante, de estos agentes los más importantes son los que causan la calidad, es decir, el equipo docente. Director y maestro tienen que compartir el propósito de mejorar la calidad, comprender que se requiere un cambio de actitudes y estar dispuestos a modificar sus actitudes y a ser consecuentes con esta decisión de cambio".

Se sostiene que para que lo anterior sea posible, es necesario que todo el equipo docente comprenda y comparta el propósito del mejoramiento y entienda bien el papel que le toca desempeñar en él. Se trata de reunir a todos los involucrados en el proceso, de manera que todos los actores, incluyendo la familia participen en torno a los objetivos comunes que tiene la escuela, cual es desarrollar un aprendizaje de calidad en los estudiantes e involucrarse en la búsqueda de soluciones de problemas comunitarios.

En una sociedad tan compleja, donde existen tanto problemas que obstaculizan el desarrollo integral de las organizaciones, y sobre todo en instituciones como la escuela, que necesita de la participación y el apoyo en la solución de problemas, se impone como dice Covey (1991:53):

"como seres verdaderamente independientes, podemos unirnos para lograr propósitos en común que beneficien a la familia, el grupo, la organización y la sociedad en su totalidad".

En otras palabras y centrándonos en el desarrollo de este trabajo, contribuir con nuestra imaginación creativa en el in- creíble proceso sinérgico a favor de la gestión escolar participativa en las Escuelas Bolivarianas.

El aporte de este artículo consiste en asumir algunas cuestiones dilemáticas sobre el nuevo modelo curricular en el sistema educativo venezolano, especialmente en las escuelas bolivarianas, con la intención de problematizar el asunto de la reforma educativa a efectos de intentar debatir sobre otros modos de gestión escolar participativa e intervención desde donde sea posible transitar la transdisciplinariedad. Ya que estos nuevos escenarios reclaman nuevas maneras de pensar la educación.

Este ámbito de la participación ha sido ampliamente dispuesto en la actual Constitución de la República Bolivariana de Venezuela (CRBV, 1999) en la cual de sus 350 artículos, 130 hacen referencia directa o indirecta de la participación relacionándola con la corresponsabilidad, solidaridad, organización de comunidades, defensa y protección de los derechos humanos y educación ciudadana.

En todo caso, la participación del pueblo en la formación, ejecución y control de la gestión pública es el medio necesario para lograr el protagonismo que garantice su completo desarrollo, tanto individual como colectivo. En este sentido, el protagonismo de los ciudadanos en la búsqueda de su bienestar individual y colectivo está inscrito en los artículos 3, 5, 112, 229 y 322 en la Carta Magna y aún en el preámbulo constitucional (1999:1) al establecer:

"Una sociedad democrática, representativa, participativa y protagónica, multiétnica y pluricultural en un estado de justicia federal y descentralizado que 
consolide los valores de la libertad, la independencia, la paz, la solidaridad, el bien común la integridad territorial, la convivencia y el imperio de la ley para ésta y las futuras generaciones, asegure el derecho a la vida, al trabajo, a la cultura, a la educación, a la justicia social y a la igualdad sin discriminación ni subordinación alguna...".

Este preámbulo sella la participación de la comunidad en la gestión escolar y legitima la gerencia participativa en educación; de allí que entre otras repercusiones insta una mayor y mejor participación de los padres en la escuela, abriéndole espacios para su libre cooperación.

Esta reflexión sobre la gestión participativa se inscribe en lo que Senge (2000:17) propone como el espacio en el cual las escuelas pueden rehacerse, revitalizarse y renovarse en forma sostenida a través de una orientación de aprendizaje. Esto significa "hacer que todos los que pertenecen al sistema expresen sus aspiraciones, tomen conciencia y desarrollen juntos sus capacidades". De ahí la importancia del involucramiento del proceso educativo de la escuela que plantea Delors (1996) al señalar que la escolarización como proceso, depende de mucho factores correlacionados con la naturaleza del centro educativo y de la comunidad, depende en mayor medida del valor que la colectividad conceda a la educación. Si ésta es muy apreciada y activamente buscada, la comunidad comparte y respalda la misión y los objetivos de la escuela.

En ese mismo orden, Fernández (2001) sostiene que para el desarrollo del dominio organizacional es necesario el desarrollo de cualidades y maneras de tra- bajo entre el personal directivo, profesores y comunidad. Sugiere dentro de estos requisitos que el liderazgo del director del centro y demás personal directivo, debe propiciar una gestión de cambio que facilite el trabajo en equipo, identificación conjuntas de los problemas y sus posibles soluciones, dentro de un marco ético y de creatividad. Esto propiciará el que la escuela y la comunidad conciban, elaboren y ejecuten el proyecto educativo de la escuela en un clima de asunción compartida.

Ahora bien, la gestión escolar se define como el proceso que busca el fortalecimiento de una cultura de participación de la comunidad, teniendo como eje central la escuela, incrementando la base democrática de la sociedad, a la vez que propicia mejores condiciones educativas para los miembros de la propia comunidad.

Así, según los planteamientos de Lanz (1998) la gestión escolar es conceptuada como el conjunto de acciones pedagógicas integradas con las gerenciales que realiza un directivo, con múltiples estrategias, estructuradas convenientemente, para influir en los sujetos del proceso educacional, que partiendo de objetivos permiten conducir un sistema escolar del estado inicial al deseado con vistas a cumplir un encargo social determinado. Su génesis es amplia, puesto que en la educación hay muchos procesos implícitos y cada uno presenta sus propias características. En la práctica la gestión escolar los integra como un todo. A los efectos de su estudio se puede enmarcar la gestión escolar en tres dimensiones: Gerencial educacional, Quehacer educacional y Valórico educativo, las cuales se entrelazan para dar cuenta de aquella. 
Reflexión transdisciplinaria acerca del deber ser de la gestión escolar en Venezuela Pulido, Sirse; Siracusa, Giuseppe y Ardiles, Arlene

En síntesis, la gestión escolar es un proceso complejo caracterizado por múltiples interrelaciones de variados nexos internos entre sus componentes, con características muy dinámicas y de diferente naturaleza que frente a las nuevas exigencias de la educación, implica múltiples y variadas formas de satisfacer sus demandas; por lo tanto reviste una intervención transdisciplinar; ya que no se trata sólo de gestionar aspectos aislados en el contexto escolar como hasta el momento se manifiesta en los sistemas educacionales, sino de tener en cuenta en la proyección, que entre los diferentes componentes del sistema escolar se dan múltiples interrelaciones y el enfoque global posibilita verlas todas en función de comprender con una mayor significación el alcance de la educación, formación integral del estudiante y consolidación del maestro.

\section{Conclusiones}

Luego de haberse presentado a grandes rasgos las características que definen los distintos enfoques epistemológicos y teóricos conceptuales, y establecida la vinculación entre ellas para la generación de una postura sociocrítica desde una perspectiva transdisciplinaria de la Gestión Escolar Participativa en las Escuelas Bolivarianas, se hacen a continuación las siguientes consideraciones:

Desde este nuevo entramado de saberes, es indudable que la descripción del mundo y de los fenómenos actuales, entre los cuales se sitúa la concepción transdisciplinar de la gestión escolar participativa de las Escuelas Bolivarianas, nos exige una nueva forma de valoración desde una configuración más amplia, con una nueva forma de pensar que reclama encontrar un nuevo paradigma capaz de interpretar la realidad actual.

Para concretar la intervención de los corresponsables del proceso educativo con miras a una verdadera participación y democratización del centro educativo es esencial una mirada reflexiva al interior de éstos para proponer espacios de participación sintonizando la gerencia con la sinergia de la complementariedad e interdependencia de los procesos educativos vitales tanto humanos como organizacionales.

En este contexto, el director como gerente institucional, en comunión con el personal docente, tiene el compromiso de impulsar la reflexión y la participación de los miembros de la comunidad educativa, propiciando el trabajo en equipo, orientar a los principales actores del hecho educativo sobre las tareas y responsabilidades implícitas en el Proyecto Educativo Nacional, lo cual conlleva concretar los lineamientos que emergen de los postulados de la Constitución de la República Bolivariana de Venezuela y delinean la gestión en las Escuelas Bolivarianas, a fin de cristalizar una legitima gestión escolar participativa en este sector de la educación venezolana.

\section{Referencias bibliográficas}

Aktouf, Omar (1998). La administración: entre Tradición y Renovación. Canadá, Gaetan Morín Editeur.

Bertalanffy, Ludwing (1984). Tendencias en la teoría general de sistema. Madrid, Alianza.

Bordieau, Pierre (1995). El oficio del sociólogo. Presupuestos epistemológicos. Buenos Aires, Siglo XXI. 
Constitución de la República Bolivariana de Venezuela (1999). Gaceta Oficial No 36.860, Venezuela.

Covey, Stephen (1991). Los siete hábitos de la gente altamente efectiva. Buenos Aires, Tema.

Delors, Jacques (1996). La educación encierra un tesoro. Paris, UNESCO.

Fernández, María (2001). Gerencia Social. Bogotá, McGraw Hill.

Gusdorf, Georges (1983). Patología del saber. [Documento en Línea]. Disponible: http://perso.club-internet.fr/nicol/ciret/ locarno/locarno.htm, [Consultado: 2010, Febrero 18].

Kliksberg, Bernando (1998). Más ética, más desarrollo. Buenos Aires, Temas

Lakatos, Imre (1982), Historia de la ciencia y sus reconstrucciones racionales. Madrid, I Tecnos.

Lanz, Carlos (1998). Currículo y Desarrollo Curricular en Las Escuelas. Caracas, Espíteme.

Limardo, Marcos (2000). La gerencia coparticipativa. Caracas, Espíteme.

Martínez, Miguel (2006). La Nueva Ciencia. México, Trillas.

Ministerio de Educación, Cultura y Deporte (2001). Proyecto Educativo Nacional. Caracas, Autor.

Ministerio de Educación, Cultura y Deporte (2000). Resolución N 751 Comunidades Educativas. Caracas, Autor.

Ministerio de Educación, Cultura y Deporte (2000). Resolución № 179 de fecha 15 de septiembre de 1999. Caracas, Autor.

Ministerio de Educación, Cultura y Deporte (1997). Proyecto Educativo Nacional (PEN). Caracas, Autor.

Ministerio de Educación, Cultura y Deporte (1996). Normativo de Escuelas Bolivarianas. Caracas, Autor.

Morín, Edgar (2003). Introducción al pensamiento complejo. Barcelona, Gedisa.
Morín, Edgar (2001)." El pensamiento complejo contra el pensamiento único". Entrevista realizada por Nelson Vallejo Gómez en Sociología y Política, México, Nueva Época, año IV, No. 8.

Nicolescu, Basarab (1998). Transdisciplinarity and complexity: Levels of reality as source of indeterminacy. Bulletin Interactif du Centre International de Recherches et Études Transdisciplinaires. [Documento en Línea]. Disponible: http://nicol.club.fr/ciret/bulletin/ b15/b15c4.htm, [Consultado: 2009, Diciembre 7].

Nicolescu, Basarab (1996). La transdisciplinariedad, una nueva visión del mundo. Paris, Ediciones Du Rocher.

Organización de las Naciones Unidas para la Educación (2005). Convenio para Mejorar la Gestión Educativa. [Documento en Línea]. Disponible: http://prensa.Mendoza.gov.ar/, [Consulta: 2009, julio 25]

Organización de las Naciones Unidas para la Educación, la Ciencia y la Cultura (2001). Informe de la UNESCO para la educación del siglo XXI. París.

Peñuela, Alejandro (2005). La transdisciplinariedad. Más allá de los conceptos, la dialéctica. Revista Electrónica de Investigación Social, $\mathrm{N}^{\circ} 2$, [Documento en Línea]. Disponible: http://dialnet.unirioja.es/ servlet/articulo?codigo $=2012$ 602, [Consulta: 2009, junio 19].

Piaget, Jean (1978). Las Estructuras Cognoscitivas. Madrid, Siglo XXI.

Ponce, Guillermo (1999). Toda la patria una escuela. Caracas, Espíteme.

Schemelkes, Sylvia (1992). Hacia una mejor calidad de nuestras escuelas. [Documento en línea]. Disponible en: http://carloscalvo.blogspirit.com/files/ schmelkes.pdf, [Consulta: 2009, junio19].

Senge, Peter (2000). La Quinta Disciplina. México, McGraw Hill. 